\title{
Dermatologic Manifestations of the LEOPARD Syndrome
}

\author{
S. Cao and A.F. Nikkels* \\ Department of Dermatology, University Hospital of Liège, Sart Tilman, Liège, Belgium
}

\begin{abstract}
The LEOPARD syndrome is an exceptional autosomal dominant genetic disease with a missense mutation of the PTPN11 gene in more than $90 \%$ of the cases. The principal clinical manifestations include extensive lentiginosis, heart conduction abnormalities, hypertrophic obstructive cardiomyopathy, ocular hypertelorism, pulmonary stenosis, genital anomalies, mental retardation, growth retardation and deafness. A woman with a LEOPARD syndrome illustrates the progressive development of melanocytic nevi. In fact, the majority of lentigines are actually melanocytic nevi. Sequential digital dermoscopy evidences progressive growth of some melanocytic lesions. The ever-increasing number of melanocytic nevi in the LEOPARD syndrome is a risk factor for melanoma and full body photography and dermoscopy are recommended for follow-up.
\end{abstract}

Keywords: LEOPARD syndrome, melanocytic nevi, dysmorphism, lentiginosis, PTPN11.

\section{INTRODUCTION}

The LEOPARD syndrome is an exceptional dominant autosomal disease (Lentiginosis, ECG conduction anomalies, Ocular hypertelorism/hypertrophic Obstructive cardiomyopathy (HOCM), Pulmonary stenosis, Abnormalities of genitalia, growth Retardation and Deafness)[1]. The first case was described in 1936 but the LEOPARD term was first used in 1969 [2]. Up to date, about 300 cases have been reported [3]. Many synonyms exist: cardiomyopathic progressive lentiginosis, multiple lentigines syndrome, cardio-cutaneous syndrome or Moynahan syndrome [1]. The LEOPARD syndrome is currently also referred to as Noonan syndrome with multiple lentigines or NSML (OMIM 151100) [3]. A woman is presented to illustrate the highly polymorphous character of this genetic syndrome, insisting in particular on the progressive evolution of the dermatologic features and the importance of repetitive total body photography and dermoscopy in the dermatologic follow-up.

\section{CASE REPORT}

A 38-years-old woman patient presented at the dermatology ward for multiple pigmented lesions. Clinical examination showed multiple variable-sized lentigines and melanocytic nevi covering the entire body. The first lentigines appeared at the age of 6 on her face and the upper back (Figs. 1, 2). At puberty, the number and size of lesions increased and they became more pigmented (Figs. 1, 2). Previous total body photography and dermoscopy never identified any suspect pigmented lesion. Several "lentigines" were previously excised as they appeared atypical but histopathology always identified benign melanocytic nevi. The patient was born at term after a pregnancy without any significant complications. Her birth weight was $4.050 \mathrm{~kg}$ and

*Address correspondence to this author at the Department of Dermatology, CHU - Sart Tilman, University of Liège, B-4000, Liège, Belgium;

Tel: +32 4366 7232; Fax: +32 4366 7234; E-mail: af.nikkels@chu.ulg.ac.be the body length $54 \mathrm{~cm}$. She did not present any neonatal problems. Nevertheless, she appeared hypotonic at birth. Rapidly, a bilateral strabismus with a congenital nystagmus was noticed. Her psychomotor development was relatively slow: walking and speech acquisition at 3 years and diurnal sphincter control at 2 years with persistent enuresis until 25 years. She has been epileptic with tonoclonic crises and absences since she was 11 . Mild mental retardation was noted with an IQ at 74. She followed specialized education from 7 to 21 years. She is able to read, write and to perform simple calculations. Currently, she lives with her mother and spends the day in a specialized occupational center. Clinical examination revealed an increased interpupillary distance. Her length was $1 \mathrm{~m} 74$ and her weight $53 \mathrm{~kg}$. Further medical history revealed asthma, anorexia, a surgical intervention for strabismus, osteoporosis and a minor chronic peripheral polyneuropathy of the limbs in relation with an autoimmune hypothyroidism. The latter was revealed by a delayed puberty. No gynecologic problem has ever been detected. There was no previous family history of lentiginosis. Her present treatment includes carbamazepine $\left(\right.$ Tegretol $\left.^{\circ}\right)$, montelukast (Singulair ${ }^{\circ}$ ), desloratadine (Aerius ${ }^{\circ}$ ), levothyroxine (Elthyrone ${ }^{\circ}$ ) and heptaminol. As she presented hypertelorism and delayed psychomotor development, a karyotype was requested in 1978 and did not detect any chromosomal abnormalities. In 1992, an autoimmune thyroiditis was evidenced with an exaggerated prolactin (PRL) reactivity to the TRH test, maybe related to her carbamazepine treatment. Later, a low ferritin level prompted oral iron supplementation. Because of persistent headaches and increased PRL level, a MRI of the sella turcica was performed in 2003 but did not reveal any abnormalities. The last cardiological exploration was performed in 2008. The ECG demonstrated a right-axis deviation and a left posterior fascicular block. Nevertheless, the whole examination was practically within the normal limits. In 2009, a hearing test didn't reveal any signs of deafness. Genetic analysis by molecular analysis did not identify any mutation in the PTPN11 and RAF1 genes. 


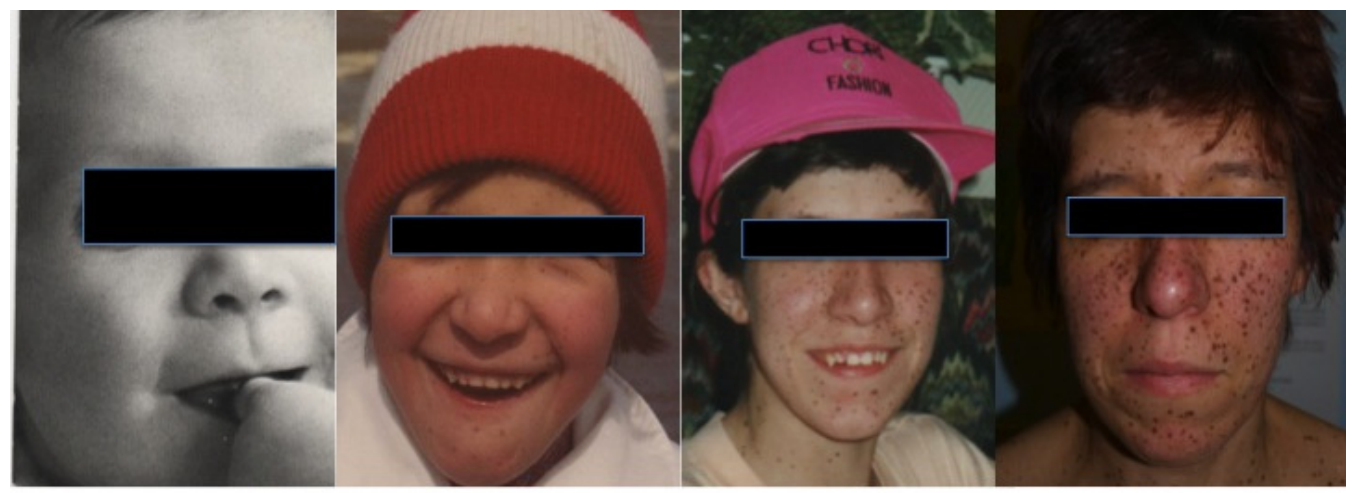
6 months
6 years
15 years
38 years

Fig. (1). Progressive appearance of the melanocytic lesions of the face.

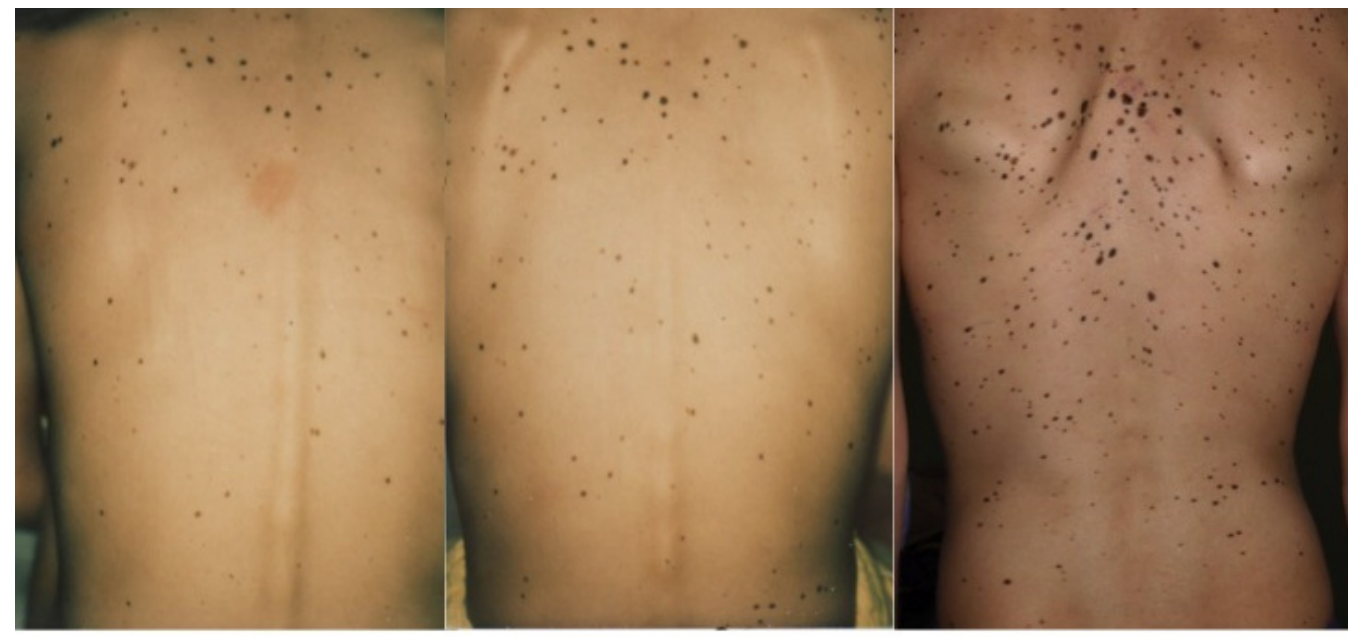

10 years

14 years

38 years

Fig. (2). Evolution of the melanocytic lesions of the back.

\section{DISCUSSION}

The LEOPARD syndrome is an exceptional disorder with an autosomal dominant transmission. The penetrance is very high and the expression highly variable. In about $90 \%$ of the cases, it is linked to a germline PTPN11 missense mutation with loss of function $[1,4]$. The major clinical features are diffuse lentiginosis, ECG abnormalities, ocular hypertelorism, hypertrophic cardiomyopathy, pulmonary stenosis, genital anomalies, retardation of growth and deafness. Additional characteristics are a facial dysmorphism, skeletal abnormalities, neurological troubles, as observed in this case, hypotonia at birth, learning disabilities with mental retardation, oculomotor defects and EEG abnormalities 「1, 5]. Rare cases of cancer, including melanoma, had been reported. Nevertheless, as total case number is so low, no particular cancer susceptibility is currently established [3].

The diagnosis of a LEOPARD syndrome requires diffuse lentiginosis and two other syndrome traits. If diffuse lentigines are absent, the presence of a first degree affected relative and three other distinct features are necessary. However the diagnosis is not always obvious, especially as some signs are lacking or only appear at an advanced age. Furthermore, it shares lots of characteristics with other genetic syndromes like the Noonan syndrome, also mediated by a PTPN11 mutation but of the gain function type [1]. Further differential diagnoses include type 1 neurofibromatosis, Costello syndrome, cardiofaciocutaneous syndrome, Peutz-Jeghers syndrome and Carney complex. All these syndromes, except the two latter, are in relation with a mutation in the RAS-MAPK signaling pathway that is involved in cellular proliferation, growth and differentiation $[6,7]$. In atypical cases of LEOPARD syndrome, a missense PTPN11 mutation confirms the diagnosis [4]. In case of negativity, a RAF1 mutation should be searched for that is present in $1 / 3$ of the patients without a PTPN11 mutation [1]. In less than 5\%, a BRAF mutation can be associated [8]. Molecular analysis provides additional interesting clinical information helping in the long-term management and follow-up of these patients. Indeed, the prevalence of cardiac conduction disorders, ventricular or left auricular hypertrophy and familial history of sudden death is significantly higher in PTPN11 mutation negative patients. On the other hand, among the PTPN11 mutations, those 
involving the exon 13 are associated with an increased risk of hypertrophic cardiomyopathy and severe cardiac complications whereas those involving exon 7 are more often linked to growth retardation and deafness compared to exon 12 mutations [1]. The presence of BRAF mutations are usually linked to moderate to severe cognitive impairment [9].

The mortality and morbidity predominantly depend on the extent of the cardiac abnormalities. A complete checkup, including rigorous clinical examination, growth parameters monitoring in children, hearing test (1/year until adulthood) and cardiological (1/year mainly when lentigines appear if none cardiac lesion was previously detected), neurological and urogenital evaluations, is highly recommended. In contrast, the management of other abnormalities does not differ from those in general population [1].

The multiple lentigines and melanocytic nevi require a regular follow-up because of their progressively increasing total number. A total UVA-UVB protection is advocated. Multiple melanocytic nevi are a significant risk factor for melanoma. As these patients steadily increase the number of lesion over lifetime, the potential risk for melanoma will theoretically increase. Until now, two cases of malignant melanoma have been reported in 300 reported cases $[3,10,11]$. Hence, a yearly total body photography [12], in addition to clinical examination and dermoscopic analysis can be very helpful for follow-up, as demonstrated in this patient. Dermoscopic follow-up with the Dermogenius ${ }^{\circ}$, a digitalized epiluminescence microscopy system clearly

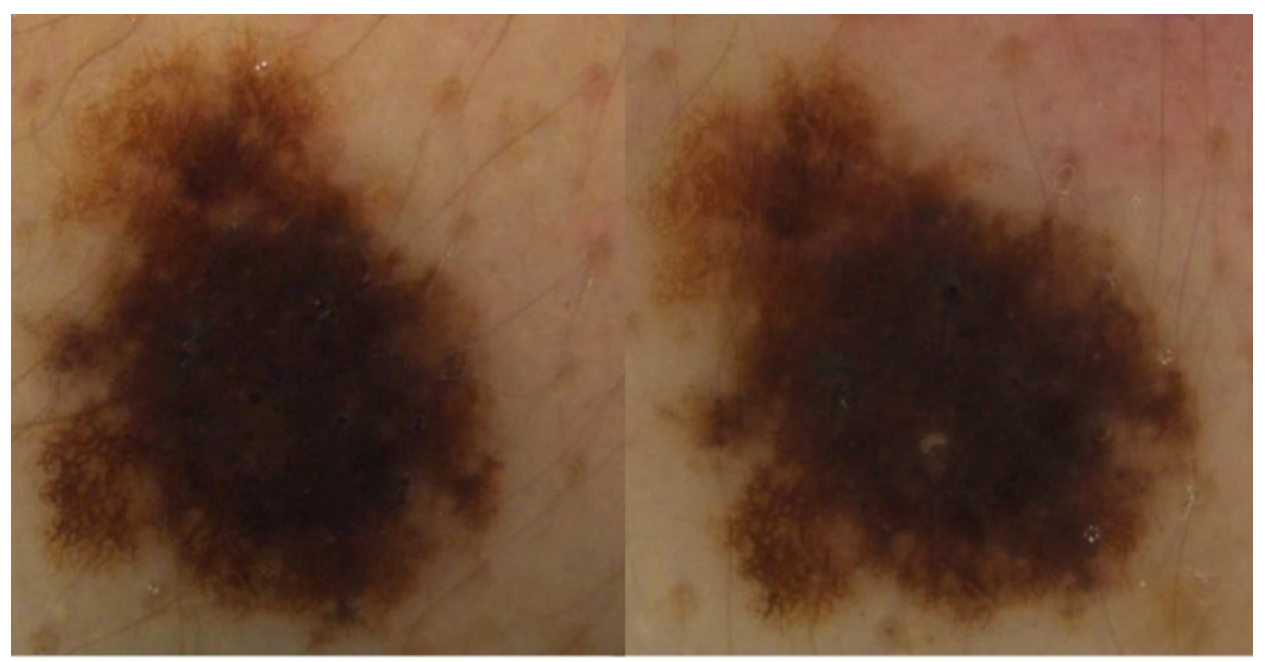

Pigmentary naevus 1 . Increase in size over one year, note peripheral radial growth.

Fig. (3). Dermoscopic evolution of a melanocytic nevus.

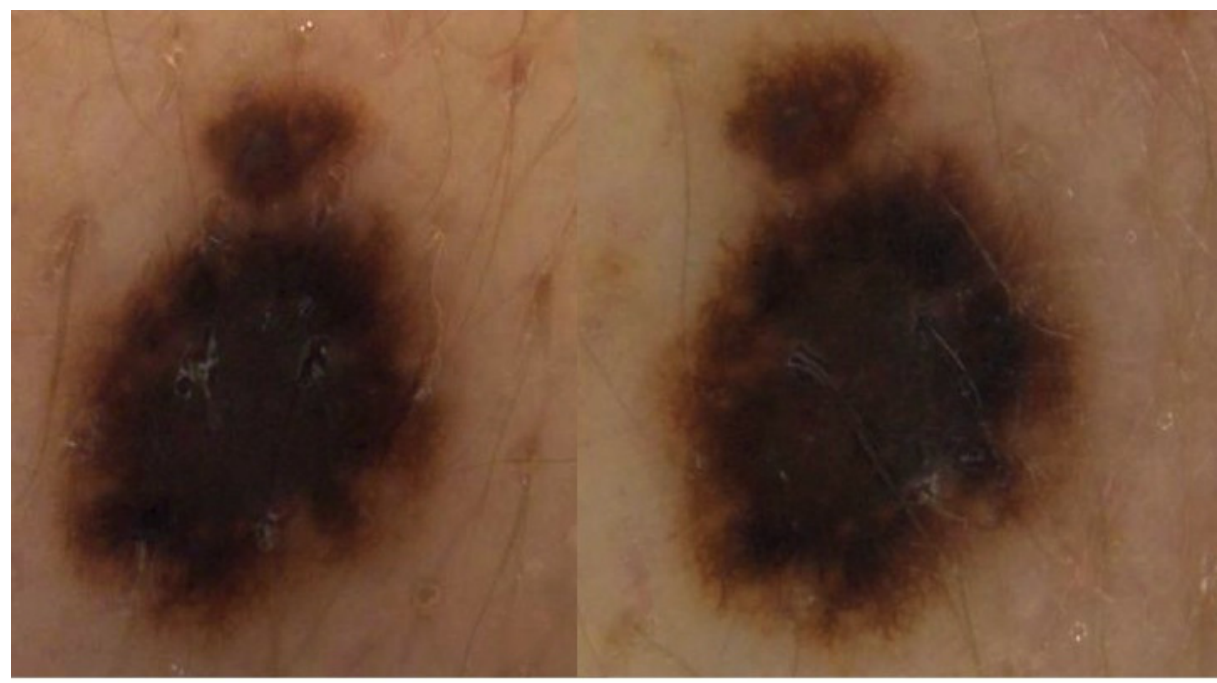

Pigmentary naevus 2. Increase in size over one year, note peripheral radial extension.

Fig. (4). Dermoscopic evolution of a melanocytic nevus. 
reveals growth in size and increase of pigmentation in certain melanocytic nevi (Figs. 3, 4). For cosmetic reasons, some patients ask for treatment of the multiple lentigines. Many treatments are available including dermabrasion, electrodessication, cryotherapy, surgical excision, chemical peels, laser or intense pulsed light. One case was successfully treated by intense pulsed light [13].

\section{CONCLUSION}

LEOPARD syndrome is an exceptional autosomal dominant disorder with high penetrance and marked variable expression. It is frequently associated with a PTPN11 missense mutation. It is principally characterized by multiple lentigines and melanocytic nevi, cardiographic conduction defects, ocular hypertelorism, pulmonary stenosis, genital abnormalities, growth retardation and deafness. Repetitive total body photography combined with a thorough clinical and dermoscopic examination should help in the surveillance of the multiple lentigines and melanocytic nevi. The term NEOPARD (multiple nevi) is proposed as it suits more adequately the dermatologic alterations.

\section{CONFLICT OF INTEREST}

The authors confirm that this article content has no conflict of interest.

\section{ACKNOWLEDGEMENTS}

Declared none.

\section{REFERENCES}

[1] Sarkozy A, Digilio MC, Dallapiccola B. Leopard syndrome. Orphanet J Rare Dis 2008; 3: 13.
[2] Gorlin RJ, Anderson RC, Blaw M. Multiple lentigines syndrome Am J Dis Child 1969; 117: 652-62.

[3] Kratz CP, Rapisuwon S, Reed H, Hasle H, Rosenberg PS. Cancer in Noonan, Costello, cardiofaciocutaneous and LEOPARD syndromes. Am J Med Genet C Semin Med Genet 2011; 157: 83-9.

[4] Kato H, Yoshida R, Tsukamoto K, et al. Familial cases of atypical clinical features genetically diagnosed as LEOPARD syndrome (multiple lentigines syndrome). Int J Dermatol 2010; 49: 1146-51.

[5] Coppin BD, Temple IK. Multiple lentigines syndrome (LEOPARD syndrome or progressive cardiomyopathic lentiginosis). J Med Genet 1997; 34: 582-6.

[6] Lodish MB, Stratakis CA. The differential diagnosis of familial lentiginosis syndromes. Fam Cancer 2011; 10: 481-90.

[7] Denayer E, Legius E. What's new in the neuro-cardio-facialcutaneous syndromes? Eur J Pediatr 2007; 166:1091-8.

[8] Gelb BD, Tartaglia M. LEOPARD Syndrome. In: Pagon RA, Bird TD, Dolan CR, Stephens K, Adam MP, Eds. Seattle (WA): Gene Reviews 1993. Gene Rev 2007; 2010: 16.

[9] Koudova M, Seemanova E, Zenker M. Novel BRAF mutation in a patient with LEOPARD syndrome and normal intelligence. Eur J Med Genet 2009; 52: 337-40.

[10] Seishima M, Mizutani Y, Shibuya Y, Arakawa C, Yoshida R, Ogata T. Malignant melanoma in a woman with LEOPARD syndrome: identification of a germline PTPN11 mutation and a somatic BRAF mutation. Br J Dermatol 2007; 157:1297-9.

[11] Jurecka W, Gebhart W, Knobler R, Schmoliner R, Möslacher H. The leopard syndrome, a cardio-cutaneous syndrome. Wien Klin Wochenschr 1983; 95: 652-6.

[12] Salerni G, Carrera C, Lovatto L, Puig-Butille JA, Badenas C, Plana E, Puig S, Malvehy J. Benefits of total body photography and digital dermatoscopy ("two-step method of digital follow-up") in the early diagnosis of melanoma in patients at high risk for melanoma. J Am Acad Dermatol. 2012; 67: e17-27.

[13] Kontoes PP, Vlachos SP, Marayiannis KV. Intense pulsed light for the treatment of lentigines in LEOPARD syndrome. Br J Plast Surg 2003; 56: 607-10. 